\title{
Ambulation three hours after elective cardiac catheterisation through the femoral artery
}

Giuseppe Steffenino, Antonio Dellavalle, Flavio Ribichini, Paolo Russo, Laura Conte, Stefania Dutto, Giuseppina Giachello, Giulietta Lice, Marilena Tomatis, Eugenio Uslenghi

\begin{abstract}
Objective-To test whether very early resumption of ambulation after femoral cardiac catheterisation is feasible and safe in patients with stable symptoms.

Design-Prospective study in a selected group of men and women undergoing elective cardiac catheterisation, with next day physical inspection.

Setting-Inpatient study.

Subjects-Two hundred consecutive ambulant patients submitted to diagnostic cardiac catheterisation through the femoral arterial route using $5 F$ catheters: a femoral right heart study was done at the same time in 40 patients $(20 \%)$.

Results-No patient had major complications during the study. Early ambulation was not allowed in two patients $(1 \%)$ because of haematoma formation immediately after sheath removal, and in seven (3\%) because of poor haemostasis or haematoma on inspection at 3 h. Early ambulation was interrupted in two patients $(1 \%)$ because of transient arterial hypotension on standing in one, and the patient's preference in the other. Of 189 patients who resumed full ambulation at 3 $h$, one $(0.5 \%)$ had a groin haematoma on discharge the next morning. Overall, haematoma $12 \mathrm{~h}$ after cardiac catheterisation was present in seven of the 200 patients initially included in the study (3.5\%). None of the 191 patients with attempted early mobilisation had signs or symptoms of vascular complications one month or later after discharge.
\end{abstract}

Conclusion-Supervised resumption of ambulation $3 \mathrm{~h}$ after uncomplicated cardiac studies with $5 F$ femoral arterial catheters is safe and feasible in most ambulant patients undergoing elective cardiac catheterisation.

(Heart 1996;75:477-480)

Keywords: coronary angiography; femoral cardiac catheterisation; early ambulation; complications

Increasing numbers of patients undergo diagnostic cardiac catheterisation. These include a growing population of stable patients needing elective angiographic and haemodynamic assessment after myocardial infarction treated with intravenous thrombolysis, or follow up studies after coronary interventions or cardiac transplantation. Early discharge after cardiac catheterisation can add to patients' satisfaction, improve the utilisation of both inpatient and outpatient facilities, and reduce costs. Early mobilisation after femoral coronary arteriography has been described. ${ }^{1-3}$ The potential for significant arterial bleeding or cardiac complications, however, remains of concern for many cardiologists, and prolonged bed rest is generally recommended after transfemoral cardiac catheterisation. This has also been current practice in our centre since 1992, and outpatients have not been allowed to resume ambulation before six hours following cardiac catheterisation. ${ }^{4}$ Our aim in this study was to evaluate, in a prospective trial, the safety of very early mobilisation after elective cardiac catheterisation with femoral arterial $5 \mathrm{~F}$ (French) catheters.

\section{Methods}

Two hundred consecutive ambulant inpatients undergoing elective cardiac catheterisation through the femoral arterial route in our institution were enrolled in the study between October 1994 and April 1995. Exclusion criteria are listed in table 1 . The established routine of our centre was followed for cardiac catheterisation. A $5 \cdot 2 \mathrm{~F}$ femoral arterial sheath and $5 \cdot 2 \mathrm{~F}$ catheters were used in all cases (Super-torque plus, Cordis: internal lumen $=$ $1.1 \mathrm{~mm}$; maximum flow rate $18 \mathrm{ml} / \mathrm{s}$ ). Catheter configurations (Judkins, Amplatz, multipurpose, mammary, NIH, and pigtail) were selected according to the patient's anatomy and the operator's preference. When necessary, a $5 \cdot 2 \mathrm{~F}$ or $8 \mathrm{~F}$ sheath was concomitantly placed in the femoral vein of the same side, and right heart catheterisation was performed with either $5 \cdot 2 \mathrm{~F}$ multipurpose or pigtail catheters, or $7 \mathrm{~F}$ thermodilution SwanGanz catheters. Heparin $5000 \mathrm{U}$ was given immediately after placement of the arterial sheath. Selective coronary arteriography was performed by hand injection of 6 to $10 \mathrm{ml}$ of ionic contrast medium (ioxaglate) at room temperature, in multiple views. Left ventriculography was performed by power injection

Table 1 Exclusion criteria

- Outpatient

- Patient not fully mobilised before catheterisation

- Previous access $<1$ month through the same femoral artery

Ongoing medication with heparin or oral anticoagulants

Platelet count below $100000 / \mathrm{ml}$

- Prothrombin time $<80 \%$ or activated partial thromboplastin time $>50 \mathrm{~s}$ thromboplastin time $>50$
Severe aortic regurgitation

- Severe aortic regurgitation

Acute angina at rest (Braunwald's grade III) ${ }^{5}$ 
(Angiomat 6000, Liebel-Flarsheim) in one or two projections as required: the rate of contrast injection ranged from 8 to $18 \mathrm{ml} / \mathrm{s}$ for total volumes of 30 to $65 \mathrm{ml}$. A Polydiagnost C2 $x$ ray system with Digital Cardiac Imaging (Philips Medical Systems) was used for imaging, with framing rates of 12.5 or $25 / \mathrm{s}$. This equipment allows automatic loop replay of digital cine runs immediately after their termination. After completion of the study, protamine sulphate was not used to reverse the effect of heparin. The sheaths were removed and the puncture site was compressed manually until satisfactory haemostasis was achieved. A disinfectant dressing was applied and secured by a tight adhesive elastic gammashaped bandage from hip to hip around the thigh. The study protocol was terminated if patients developed cardiac symptoms during the procedure or if a haematoma (localised swelling around the puncture site of $4 \mathrm{~cm}$ or more in diameter) had occurred by that time (early dropout).

Patients remained at rest in bed for $3 \mathrm{~h}$ until they were seen by one of the nurses of the catheterisation laboratory. Before asking the patient to get up and walk the vital signs were observed, the bandage was removed, the puncture site was inspected, and an electrocardiogram was recorded. The study protocol was terminated (late dropout) if local haemostasis was not satisfactory, or if haematoma, uncontrolled arterial hypertension (systolic blood pressure $>180 \mathrm{~mm} \mathrm{Hg}$ ), cardiac symptoms, or ischaemic changes on the electrocardiogram were present at that time.

Early ambulation was supervised by the nurse during 10-15 $\mathrm{min}$; it was interrupted (interrupted ambulation) and bed rest was resumed if there was severe vasovagal hypotension on standing, or if the patient preferred this for whatever reason. After early ambulation under supervision the patient's activities in the ward were unlimited (unrestricted ambulation). All patients were seen by one of the cardiologists of the cardiac catheterisation laboratory the next day, $12 \mathrm{~h}$ or later after resumption of ambulation. Physical inspection of the patient was performed, with palpation and auscultation of the puncture site and femoral pulses, and an electrocardiogram was recorded.

Table 2 Clinical characteristics, indications for cardiac catheterisation, and diagnosis in the study patients $(n=200)$

\begin{tabular}{lc}
\hline Age (years) & $61($ SD 10$)$, range $17-84$ \\
Female & $52(26 \cdot 0 \%)$ \\
Recent $\mathrm{MI}(>10<60 \mathrm{~d})$ & $60(30 \cdot 0 \%)$ \\
Ongoing treatment with antiplatelet agents & $130(65 \cdot 0 \%)$ \\
Indications for $C C$ & \\
$\quad$ Suspected or known native CAD & $164(82 \cdot 0 \%)$ \\
$\quad$ Valvar heart disease & $32(16 \cdot 0 \%)$ \\
$\quad$ Congestive heart failure & $4(2 \cdot 0 \%)$ \\
Diagnosis at CC & \\
Non-significant coronary lesions & $10(5 \cdot 0 \%)$ \\
One vessel CAD & $69(34 \cdot 5 \%)$ \\
Multivessel CAD & $81(40.5 \%)$ \\
Left main disease & $3(1 \cdot 5 \%)$ \\
Myocardial disease & $5(2 \cdot 5 \%)$ \\
Mitral valve disease & $11(5 \cdot 5 \%)$ \\
Aortic valve disease & $21(10 \cdot 5 \%)$ \\
LVEF (\%) & $52(\mathrm{SD} 10)$, range $25-72$ \\
\hline
\end{tabular}

$\mathrm{CAD}$, coronary artery disease; $\mathrm{CC}$, cardiac catheterisation; $\mathrm{LVEF}$, left ventricular ejection fraction; MI, myocardial infraction.
Data were collected prospectively for all patients initially included in the study. The following study variables were considered: major complications, included myocardial infarction, angina, ischaemic ECG changes, vascular injury requiring blood transfusion or surgical consultation; minor complications, included vasovagal event, and painful subcutaneous blood extravasation or haematoma $>4 \mathrm{~cm}$ in diameter.

Late follow up data for all patients with early mobilisation were obtained after completion of the study, to detect possible vascular complications at the access site. The referring physician, or primary physician of each patient, was required to provide detailed information about the status of the femoral access site on inspection. Data were collected during a second telephone interview some weeks later, to allow for either retrieval of medical records or a new accurate examination of the patient. Whenever the referring or primary physician had not seen the patient one month or later after cardiac catheterisation, or was unable to provide specific information as required, the patient was contacted by a cardiologist by telephone; questions were asked about pain, swelling, or abnormalities observed at the vascular access site, and numbness or pain in the leg during ambulation.

The research protocol was approved by the ethics committee of our hospital and the informed consent of the subjects was obtained in all cases.

\section{Results}

The clinical characteristics, indications for cardiac catheterisation, and diagnoses are summarised in table 2 . The study patients represented about $25 \%$ of all patients who underwent diagnostic cardiac catheterisation in the study period in our centre. The admission criteria (table 1) were not met by 597 patients: 125 had elective cardiac catheterisation performed on a day hospital basis ( $8 \mathrm{~h}$ hospital stay), and 238 were brought by ambulance to our centre from nearby hospitals immediately before the procedure (elective) and taken back 1-3 $\mathrm{h}$ after it; in neither case would direct inspection of the femoral wound have been possible the next morning. Acute unstable angina or recent myocardial infarction was the reason for performing urgent coronary angiography in 205 patients during their admission to this or other hospitals; these patients had not been mobilised before catheterisation, and many were receiving intravenous heparin. Finally, early mobilisation was not considered in 29 patients with severe valvar disease, uncompensated heart failure, or ongoing oral anticoagulation.

Procedural variables are listed in table 3 . In no case did a larger catheter have to be substituted for the standard $5 \cdot 2 \mathrm{~F}$ catheters because of poor manoeuvrability, whiplash/recoil, or unsatisfactory coronary opacification, and images of acceptable diagnostic quality were obtained in all patients. 
Table 3 Procedural variables in the study patients $(n=200)$, values are $n(\%)$ or mean $(S D)$, range

\begin{tabular}{lc}
\hline Left and right heart study & $40(20 \%)$ \\
Retrograde catheterisation of SAV & $21(10 \cdot 5 \%)$ \\
Fluoroscopy time (min) & $5 \cdot 5(4), 1 \cdot 2-23 \cdot 7$ \\
Film length (m) & $31(5), 50-15$ \\
No of cine runs & $9(3), 4-15$ \\
Contrast volume (ml) & $150(20), 50-250$ \\
Change of catheter configuration & $16(8 \%)$ \\
Arterial time (min) & $37(16), 13-90$ \\
Compression time (min) & $12(3), 6-21$ \\
Immobilisation in bed (min) & $178(10), 165-196$ \\
\hline
\end{tabular}

SAV, stenotic aortic valve.

Table 4 Complications after cardiac catheterisation

\begin{tabular}{lc}
\hline Number of patients enrolled & 200 \\
Early dropout & 2 \\
Haematoma & $2(1 \%)$ \\
Late dropout & 7 \\
Unsatisfactory haemostasis & 3 \\
Haematoma & $4(2 \%)$ \\
Interrupted ambulation & 2 \\
Severe vasovagal hypotension & 1 \\
Patients preference & 1 \\
Unrestricted ambulation & 189 \\
Haematoma & $1(0.5 \%)$ \\
Total major complications & 0 \\
Total minor complications & 8 \\
Vasovagal & 1 \\
Haematoma & $7(3.5 \%)$ \\
\hline
\end{tabular}

ECG, electrocardiogram.

A detailed account of dropouts and complications is given in table 4 . No patient had major complications throughout the study. Nausea was a complaint during coronary angiography in 14 patients, and vomiting in two-none required medication Unsatisfactory haemostasis was a cause for late dropout in three patients: oozing of blood from the puncture site was observed upon removal of the bandage at $3 \mathrm{~h}$. The bandage was left in place, and rest in bed was continued for an additional 5-12 h. None of these patients developed haematoma. In 191 patients, mobilisation was attempted within 15 minutes of the target time, and was interrupted in two. Of 189 patients with unrestricted ambulation, one showed haematoma the next morning. Overall, haematoma was present in seven $(3.5 \%)$ of patients initially included in the study. A small, non-tender subcutaneous extravasation of blood (bruise) without swelling was observed in 10 patients (5\%).

Follow up data at 5 (SD 3) months (range 1 to 8 months) after cardiac catheterisation were obtained for all 191 patients with attempted early mobilisation. In 180 cases the physician had seen the study patient at least once one month or later after cardiac catheterisation, and could provide information from inspection of the vascular access site; in no case were vascular complications observed. Haematoma had disappeared without sequelae about two weeks after cardiac catheterisation in the only patient showing this complication at discharge. A direct telephone interview was attempted with 11 patients: one had died following coronary artery bypass surgery 10 days after cardiac catheterisation; the remaining 10 had no signs or symptoms of vascular complications-in one case following a coronary angioplasty procedure performed about $7 \mathrm{~d}$ after diagnostic cardiac catheterisation through the same femoral artery.

\section{Discussion}

Although the performance of $5 \mathrm{~F}$ catheters for cardiac catheterisation appeared unsatisfactory in many early trials, ${ }^{267}$ these catheters are nowadays used for cardiac catheterisation in many centres by both the brachial ${ }^{89}$ and the femoral ${ }^{10}$ route, because of technical improvements in catheter quality, $x$ ray imaging equipment, and operator experience. Large lumen $5 \cdot 2 \mathrm{~F}$ catheters have been routinely used for percutaneous femoral and brachial arterial cardiac catheterisation in our laboratory in over 3000 patients since 1992, with only exceptional need $(0.4 \%$ of patients) for $7 \mathrm{~F}$ catheters due to peripheral vessel tortuosity, or poor coronary opacification in individual patients with severe aortic regurgitation. The percutaneous brachial route is not used electively in our centre since it is less operator friendly in our angiographic suite, and greater radiation exposure for both the patient and the operator is reported with this approach. ${ }^{89}$ In all patients in this study, images of diagnostic quality could be obtained with $5 \cdot 2 \mathrm{~F}$ catheters, at the price of one additional catheter exchange in $8 \%$ of cases. Our mean fluoroscopy time $(5 \cdot 5$ $\mathrm{min}$ ), however, compares favourably with other studies of both femoral (mean $6.6 \mathrm{~min}$ ), ${ }^{3}$ and brachial (mean $8.7 \mathrm{~min})^{7}$ cardiac catheterisation using $5 \mathrm{~F}$ catheters, despite a right heart study and retrograde catheterisation of a stenotic aortic valve being performed in $20 \%$ and $11 \%$ of our patients, respectively.

Elective outpatient $7 \mathrm{~F}$ or $8 \mathrm{~F}$ femoral cardiac catheterisation with ambulation at six hours has been reported to be complicated by acute myocardial infarction in $1.6 \%$ of patients, and haematoma or other vascular problems in $14 \%$ of patients. ${ }^{1}$ More recent studies support the safety of five hour, ${ }^{10}$ four hour, ${ }^{11}$ and three hour ${ }^{2}$ immobilisation after femoral cardiac catheterisation with $5 \mathrm{~F}$ or $6 \mathrm{~F}$ catheters, with no major complications after the procedure, and a $1 \%, 1.5 \%$, and $10 \%$ incidence, respectively, of haematoma. In one of these reports, however, there were large deviations (range one to nine hours) from the target ambulation time. ${ }^{2}$ Right heart catheterisation as a part of the procedure was not described in these studies, protamine was routinely used for heparin reversal before clamp compression, and no direct inspection was performed the next day. Only inpatients were included in our study, no protamine was used, and a careful physical inspection was performed before discharge in all cases.

Our minor acute vascular complication rate (3.5\% overall) compares favourably with the $10 \%$ reported by Kern et al. ${ }^{2}$ This may partly be due to the single centre design of our trial, with strict selection of patients and manual femoral compression in all cases. Our finding that most haematomas occur early rather than late is in agreement with previous reports. ${ }^{23}$ In fact, of seven minor vascular complications present at discharge, only one could possibly 
have been due to early ambulation, and have been avoided by conventional prolonged bed rest.

Femoral arteriovenous fistula or pseudoaneurysm formation may be of concern following early mobilisation after femoral catheterisation. These late ( $>$ two weeks) vascular sequelae are reported to occur in $<0.5 \%$ of cases with the use of larger catheters. ${ }^{12}$ These complications almost invariably cause symptoms, and come to the attention of the general practitioner or referring physician. Follow up information at one month or later after cardiac catheterisation, from either inspection by a physician or a direct telephone interview with the patient, had the potential to detect severe vascular problems occurring late after cardiac catheterisation; this was not the case in any of the study patients.

In conclusion, our data confirm that supervised resumption of ambulation three hours after an uncomplicated cardiac study with $5 \mathrm{~F}$ arterial catheters is safe and can be applied to most ambulant patients undergoing elective cardiac catheterisation in stable conditions. Minor acute vascular complications should not be of concern in this setting, since their incidence is very low and is not appreciably increased by early mobilisation itself. The concomitant performance of a femoral right heart study does not appear to increase the risk of early vascular complications in these patients. Late sequelae at the vascular access site are infrequent, and were not likely to be encountered in our patients because of the limited sample size of the study; although conclusions cannot be drawn as to the incidence of femoral arteriovenous fistula or pseudoaneurysm after early mobilisation following femoral catheterisation with $5 \mathrm{~F}$ catheters, these complications were not observed in any of our patients.

It may be of interest that, on the basis of this study, supervised resumption of ambulation in now being implemented whenever appropriate by the nurses on our ward. This appears to improve the comfort of patients, especially the elderly, and to reduce the need for bed assistance by the personnel. The shorter rest in bed has not increased the number of outpatient cardiac catheterisation procedures in our centre, where a busy day hospital is run by the personnel of the cardiac laboratory. It allows us, however, to discharge in the early afternoon most day hospital patients admitted early in the morning. Using the same bed for two consecutive patients the same day would thus become possible, if required.

We thank Prof John Grist for his kind help in preparing this manuscript.

1 Block PC, Ockene I, Goldberg RJ, Butterly J, Block E, Degon C, et al. A prospective randomized trial of outpatient versus inpatient cardiac catheterization. $N$ Engl $\mathcal{F}$ Med 1988;319:1251-5.

2 Kern MJ, Cohen M, Talley D, Litvack F, Serota $H$, Aguirre F, et al. Early ambulation after 5 French diagnostic cardiac catheterization: results of a multicenter trial. $\mathcal{f}$ Am Coll Cardiol 1990;15:1475-83.

3 Lau KW, Tan A, Koh TH, Koo CC, Quek S, Ng A, et al. Early ambulation following diagnostic 7-French cardiac catheterization: a prospective randomized trial. Cathet Cardiovasc Diagn 1993;28:34-8.

4 Dellavalle A, Steffenino G, Ribichini F, Conte L, Dutto S, Giachello $G$, et al. Emodinamica diagnostica in regime di day-hospital. G Ital Cardiol 1993;23:973-7.

5 Braunwald E. Unstable angina. A classification. Circulation 1989;80:410-3.

6 Brown RIG, MacDonald AC. Use of 5 French catheters for cardiac catheterization and coronary angiography. A critcardiac catheterization and coronary angiography. A

7 Molajo AO, Ward C, Bray CL, Dobson D. Comparison of the performance of superflow $(5 \mathrm{~F})$ and conventional $8 \mathrm{~F}$ catheters for cardiac catheterization by the femoral route. Cathet Cardiovasc Diagn 1987;13:275-6.

8 Bush CA, VanFossen DB, Kolibash AJ, Magorien RD, Bacon JP, Ansel GM, et al. Cardiac catheterization and coronary angiography using 5 French preformed (Judkins) catheters from the percutaneous right brachial approach: a comparative analysis with the femoral approach. Cathet Cardiovasc Diagn 1993;29:267-72

9 Lupon-Roses J, Domingo E, Angel J, Anivarro I, SolerSoler J. Percutaneous right brachial artery approach with $5 \mathrm{~F}$ catheters for studying coronary artery disease. Cathet Cardiovasc Diagn 1991;22:47-51.

0 Slack JD, Higgins B, Pinto R. Use of 5 French catheters for elective outpatient catheterization: minimal early and late access site bleeding despite early ambulation protocol [abstr]. Circulation 1994;90:I-488.

11 Nagtegaal EM, deRidder TH, Schalij MJ, Buis B. Fourhour immobilization period following diagnostic cardiac catheterization [abstr]. Eur Heart $f$ 1994;15(abstr suppl): 225.

12 Johnson LW, Lozner EC, Johnson S, et al and the Registry Committee for Cardiac Angiography and Interventions. Registry of the Society for Cardiac Angiography the Registry of the Society for Cardiac Angiography and Cardiovasc Diagn 1989;17:5-10. 